\title{
Elevated L1CAM expression in precursor lesions and primary and metastastic tissues of pancreatic ductal adenocarcinoma
}

\author{
FRANK BERGMANN $^{1}$, FRAUKE WANDSCHNEIDER ${ }^{3}$, BENCE SIPOS $^{5}$, GERHARD MOLDENHAUER $^{6}$, \\ BODO SCHNIEWIND ${ }^{4}$, THILO WELSCH ${ }^{2}$, PETER SCHIRRMACHER ${ }^{1}$, GÜNTER KLÖPPEL ${ }^{7}$, \\ PETER ALTEVOGT $^{6}$, HEINER SCHÄFER ${ }^{3}$ and SUSANNE SEBENS MÜERKÖSTER ${ }^{3,8}$
}

\begin{abstract}
${ }^{1}$ Institute of Pathology and ${ }^{2}$ Department of General Surgery, University of Heidelberg, Heidelberg; ${ }^{3}$ Department of Internal Medicine 1, Laboratory of Molecular Gastroenterology and Hepatology and ${ }^{8}$ Institute of Experimental Medicine, Christian-Albrechts-University Kiel, Kiel; ${ }^{4}$ Clinic of General Surgery and Thoracic Surgery, UKSH-Campus Kiel, Kiel;

${ }^{5}$ Department of Pathology, University of Tübingen, Tübingen; ${ }^{6}$ Division of Translational Immunology D015, German

Cancer Research Center, Heidelberg; ${ }^{7}$ Department of Pathology, Technische Universität München, Munich, Germany
\end{abstract}

Received May 10, 2010; Accepted June 14, 2010

DOI: 10.3892/or_00000936

\begin{abstract}
The promigratory molecule L1CAM is overexpressed in various tumors, often representing an unfavorable prognostic marker. Recently, we identified L1CAM expression in pancreatic ductal adenocarcinoma (PDAC) cells accounting for chemoresistance and increased cell migration. Thus, the present study aims at further elucidating the role of L1CAM in a larger cohort of PDAC specimens including precursor lesions and metastasis. L1CAM expression was determined by immunohistochemistry in tissues of 123 patients including tissues of 110 primary PDACs, 15 lymph node metastases and 14 liver metastases. The immunohistochemical analyses revealed L1CAM expression in $92.7 \%$ of primary PDACs, $80 \%$ of lymph node metastases and $100 \%$ of liver metastases. Furthermore, we have investigated PDAC precursors, pancreatic intraepithelial neoplasia (PanIN) lesions, revealing a significant increase of L1CAM expression with the PanIN grade (6.4 and 6.8\% in PanIN 1A and B, 35\% in PanIN 2 and 20\% in PanIN 3). The elevated expression of L1CAM already found in PanINs points to a role of L1CAM quite early in tumorigenesis of PDAC. Furthermore, its broad expression in primary tumors as well as in metastases of
\end{abstract}

Correspondence to: Dr Susanne Sebens Müerköster, Institute of Experimental Medicine, c/o Department of Internal Medicine 1, UKHS-Campus Kiel, Arnold-Heller-Str. 3, Haus 6, D-24105 Kiel, Germany

E-mail: mueerkoe@1med.uni-kiel.de

Abbreviations: PDAC, pancreatic ductal adenocarcinoma; PanIN, pancreatic intraepithelial neoplasia

Key words: pancreatic ductal adenocarcinoma, L1CAM, CD171, metastases, therapeutical target
PDAC patients provide a rationale to further explore the value of L1CAM as a therapeutic target in the treatment of this highly malignant tumor.

\section{Introduction}

Pancreatic ductal adenocarcinoma (PDAC) is a highly malignant tumor characterized by rapid tumor progression and early metastatic spread $(1,2)$. In Western countries, PDAC is 4th in the rank order of fatal tumor diseases with an overall 5-year survival rate $<2 \%$, exhibiting a still increasing prevalence (3). Displaying a largely symptomless progression, PDAC is mostly diagnosed in an advanced stage with widespread metastasis. Accordingly, for the majority of the patients no option for a curative surgical resection exists at the time of diagnosis. Furthermore, conventional chemotherapies generally prove to be poorly effective due to the pre-existing (innate) or acquired resistance of PDAC cells towards chemotherapeutic drugs $(4,5)$. Thus, there is an urgent need to define new molecular tumor markers of PDAC to improve diagnostic procedures and therapeutic options.

The promigratory molecule L1CAM (CD171) is a 200- to $220-\mathrm{kD}$ glycoprotein and a member of the immunoglobulin superfamily. It consists of six immunoglobulin-like domains at the amino terminal end of the molecule followed by five fibronectin type III homologous repeats, a single transmembrane region and a short intracellular domain (6). Initially, L1CAM has been detected in neuronal cells (7), but in recent years it has been found in an increasing number of tumors, among others in malignant melanoma, glioma, ovarian and colon cancer, and gastrointestinal stromal tumors (8-12). In several tumor entities, high L1CAM expression was associated with poor prognosis and short survival times $(11,13)$. Beside its cell surface localization, L1CAM can also be cleaved by various proteases, i.e., the matrix metalloproteinases ADAM10 and ADAM17 or by $\gamma$-secretases $(14,15)$. Soluble L1CAM has been reported to be important for migration of neuronal as well as of tumor cells $(14,15)$, 
and several studies support a role for L1CAM in tumor growth in vivo (16) as well as tumor cell invasion and metastasis of various cancers $(8,9,15)$.

Under physiological conditions L1CAM expression within the pancreas has been shown to be restricted to neuronal structures, while the acinar parenchyma and the pancreatic ducts do not express L1CAM (17-19). In pancreatic disease, L1CAM has been shown to be expressed in poorly-differentiated pancreatic endocrine carcinomas and in anaplastic pancreatic cancer $(4,20)$. In PDAC, reported findings on L1CAM expression have been controversial $(17,22)$. However, at least in a small series of tumors L1CAM expression has been detected in up to $80 \%$ of the analysed tumor samples (17) as well as in various PDAC derived cell lines, essentially contributing to a chemoresistant and migratory phenotype $(17,18,23)$. Furthermore, we could show that L1CAM is already expressed in non-neoplastic ductal cells in chronic pancreatitis (18). Accordingly, in vitro simulation of an activated, stroma-rich environment, comparable to the fibrosis in chronic pancreatitis, revealed an enhanced L1CAM expression in the human pancreatic ductal epithelial cell line (HPDE) when cultured in the presence of pancreatic myofibroblasts (18). Altogether, these data point to a crucial role of L1CAM in the tumorigenesis of PDAC. Thus, the aim of this study was to further evaluate the L1CAM expression in an extended series of PDAC, including not only primary tumors, lymph node and hepatic metastases from PDAC patients but also precursor lesions, pancreatic intraepithelial neoplasia (PanIN), to substantiate its putative role in the progression of PDAC.

\section{Materials and methods}

Patients. According to protocols approved by the ethics committees of the University of Kiel and the University of Heidelberg, tumor specimens and clinical data were obtained from 123 patients who underwent surgery for pancreatic cancer and/or metastases of pancreatic cancer in the Clinic of General Surgery and Thoracic Surgery of the University Kiel or the Department of General Surgery of the University of Heidelberg between 1997 and 2007.

The specimens included 110 primary pancreatic ductal adenocarcinomas, 15 regional lymph node metastases (with corresponding primaries available in all cases) and 14 liver metastases (with corresponding primary available in one case). The diagnoses were established according to the World Health Organisation classification of tumors (24).

Immunohistochemistry. For immunohistochemical analyses, 4- $\mu \mathrm{m}$ sections were obtained from formalin-fixed and paraffinembedded tumor specimens. Furthermore, for the analyses of pancreatic intraepithelial neoplasia lesions, multitissue arrays were designed as described elsewhere $(25,26)$. Sections obtained from both paraffin blocks and multitissue arrays were further processed as previously described $(17,27)$. Briefly, the primary antibody (clone L1-14.10) was used at a concentration of $10 \mu \mathrm{g} / \mathrm{ml}$ and incubated overnight. Detection was performed using the avidin-biotin complex method. The immunohistochemical stainings were evaluated according to the following scheme: -, negative staining in all tumor cells;
+ , positive staining in up to $20 \%$ of tumor cells; ++ , positive staining in up to $50 \%$ of tumor cells; +++ , positive staining in $>50 \%$ of tumor cells. Positively stained nerves served as intrinsic positive controls.

Statistical analysis. Correlations between the expression of L1CAM and stage (pN0 vs. pN1), grade (G1-3), and survival, as well as the grade of PanIN lesions were tested using the Mann-Whitney test, the Kruskal-Wallis test and the log-rank test, respectively. A p-value $<0.05$ was considered statistically significant.

\section{Results}

Clinicopathological findings. The present series included 123 patients, 64 women and 59 men. At the time of surgery, patients were aged 37-88 years (mean 63.4 years).

As summarized in Table I, primary tumors were obtained from 110 patients (58 women and 52 men). These tumors were located in the pancreatic head $(n=83)$, the head and the body $(n=4)$, the body $(n=7)$, the body and tail $(n=6)$, the pancreatic tail $(n=9)$ or the entire pancreas $(n=1)$. The tumor sizes were $1.2-11 \mathrm{~cm}$ in largest diameter (mean $3.6 \mathrm{~cm}$ ). According to the location and local extent of the masses, tumors were surgically removed performing a pylorus preserving Whipple procedure $(n=76)$, a classic Whipple procedure $(n=15)$, a pancreatic left resection $(n=15)$ or a pylorus preserving total pancreatectomy $(n=4)$. Histopathological examination revealed 9 well-differentiated pancreatic ductal adenocarcinomas (PDAC), 66 moderately-differentiated PDAC and 35 poorly-differentiated PDAC. According to the local tumor extent, the masses were staged as pT3 $(n=108)$ or pT4 $(n=2) .95$ patients presented regional lymph node metastases (pN1). Distant spread was histologically detected in 5 patients (non-regional lymph nodes or liver), while 105 patients clinically displayed no evidence for distant metastases. Follow-up information could be obtained for 98 of the 110 patients. At the latest follow-up, 17 patients were alive after time periods between 3 and 64 months (average 20 months) while 81 patients had died of disease after time periods between 2 and 39 months, resulting in a median survival of 15.2 months.

All 15 immunohistochemically investigated lymph node metastases were obtained from operation specimens of the above listed primary tumors (Table II). Liver metastases were obtained from 14 patients ( 7 woman and 7 men), aged between 47 and 76 years (mean 64.5 years). The corresponding primary was available in one case of the present series (Table II). Of seven patients with available follow-up, 6 had died of disease after time periods between 3 and 36 months while one patient was alive after 29 months of followup (median survival 3 months).

Immunohistochemical findings. As mentioned above, under physiological conditions the expression of L1CAM in the pancreas is restricted to nerve fibre structures, which also showed strong staining in tissues of the present series, thus serving as positive internal staining control (Fig. 1A). Within the PDAC, positivity for L1CAM was detected in 102 of 110 primary tumors $(92.7 \%)$. As shown in Table I, 24 tumors 
Table I. Clinicopathological findings and L1CAM expression in primary PDAC tissues.

\begin{tabular}{|c|c|c|c|c|c|}
\hline & \multirow[b]{2}{*}{ Total } & \multicolumn{2}{|c|}{ L1CAM 'low' } & \multicolumn{2}{|c|}{ L1CAM 'high’ } \\
\hline & & L1CAM - & L1CAM + & L1CAM ++ & L1CAM +++ \\
\hline $\mathrm{n}$ & 110 & 8 & 24 & 26 & 52 \\
\hline \multicolumn{6}{|l|}{ Gender } \\
\hline Female & 58 & 4 & 13 & 13 & 28 \\
\hline Male & 52 & 4 & 11 & 13 & 24 \\
\hline $\begin{array}{l}\text { Age (average) } \\
\text { (years) }\end{array}$ & $37-88(63.2)$ & $48-88(65.5)$ & $38-79(60.0)$ & $50-82(65.1)$ & $37-79(63.5)$ \\
\hline \multicolumn{6}{|l|}{ Location } \\
\hline Head & 83 & 7 & 19 & 18 & 39 \\
\hline Body & 7 & 1 & 2 & 2 & 2 \\
\hline Head and body & 4 & & 2 & 1 & 2 \\
\hline Body and tail & 6 & & 1 & & 4 \\
\hline Tail & 9 & & & 5 & 4 \\
\hline Head to tail & 1 & & & & 1 \\
\hline $\begin{array}{l}\text { Tumor size } \\
\text { (average) }(\mathrm{cm})\end{array}$ & $2.0-11.0(3.6)$ & $2.5-4.5(3.3)$ & $2.5-7.0(3.3)$ & $2.0-5.0(3.3)$ & $2.0-11.0(3.8)$ \\
\hline \multicolumn{6}{|l|}{$\mathrm{T}$} \\
\hline pT3 & 108 & 8 & 22 & 26 & 52 \\
\hline pT4 & 2 & & 2 & & \\
\hline \multicolumn{6}{|l|}{$\mathrm{N}$} \\
\hline pNO & 15 & 2 & 1 & 5 & 7 \\
\hline $\mathrm{pN} 1$ & 95 & 6 & 23 & 21 & 45 \\
\hline \multicolumn{6}{|l|}{ M } \\
\hline M0 & 105 & 8 & 24 & 22 & 51 \\
\hline pM1 & 5 & & & 4 & 1 \\
\hline \multicolumn{6}{|l|}{ G } \\
\hline G1 & 10 & 6 & 4 & 4 & 2 \\
\hline $\mathrm{G} 2$ & 65 & 2 & 12 & 13 & 34 \\
\hline G3 & 35 & & 8 & 9 & 16 \\
\hline \multicolumn{6}{|l|}{ Follow-up } \\
\hline D.O.D. & 81 & 6 & 15 & 16 & 44 \\
\hline Alive & 17 & 2 & 5 & 4 & 6 \\
\hline n.i. & 12 & & 4 & 6 & 2 \\
\hline $\begin{array}{l}\text { Median survival } \\
\text { (months) }\end{array}$ & 15.2 & & & & \\
\hline
\end{tabular}

D.O.D., died of disease; n.i., no information available; -, negative staining in all tumor cells; +, positive staining in up to $20 \%$ of tumor cells; ++ , positive staining in up to $50 \%$ of tumor cells; +++ , positive staining in $>50 \%$ of tumor cells.

displayed immunoreactivity in up to $20 \%$ of the tumor cells, 26 tumors showed immunoreactivity in up to $50 \%$ and 52 tumors revealed positivity in $>50 \%$ of the tumor cells. In Fig. 1B, a representative image is shown of strong L1CAM expression in carcinoma cells of primary PDAC.
In the 15 lymph node metastases, L1CAM expression was detected in 12 cases $(80.0 \%)$. In detail (Table II), one tissue sample showed immunoreactivity in up to $20 \%$ of the tumor cells, 6 tissue samples in up to $50 \%$ and 5 tissue samples in $>50 \%$ of the tumor cells, as exemplarily demonstrated in 
Table II. Clinicopathological findings and L1CAM expression in lymph node and liver metastases.

\begin{tabular}{|c|c|c|c|c|c|}
\hline \multirow[b]{2}{*}{ Patient } & \multirow[b]{2}{*}{$\begin{array}{c}\text { Gender/ } \\
\text { Age (years) }\end{array}$} & \multirow[b]{2}{*}{$\begin{array}{l}\text { Location of } \\
\text { metastasis }\end{array}$} & \multirow[b]{2}{*}{$\begin{array}{l}\text { Follow-up } \\
\text { (months) }\end{array}$} & \multicolumn{2}{|c|}{ L1CAM reactivity } \\
\hline & & & & Metastasis & $\begin{array}{l}\text { Corresponding primary } \\
\quad \text { (if available) }\end{array}$ \\
\hline 1 & M/66 & Lymph node & D.O.D. (3) & ++ & ++ \\
\hline 2 & $\mathrm{~F} / 57$ & Lymph node & D.O.D.(13) & ++ & - \\
\hline 3 & $\mathrm{~F} / 79$ & Lymph node & D.O.D. (7) & +++ & +++ \\
\hline 4 & $\mathrm{M} / 73$ & Lymph node & D.O.D.(20) & +++ & +++ \\
\hline 5 & $\mathrm{~F} / 58$ & Lymph node & D.O.D. (9) & +++ & ++ \\
\hline 6 & $\mathrm{~F} / 75$ & Lymph node & D.O.D. (8) & +++ & +++ \\
\hline 7 & $\mathrm{~F} / 77$ & Lymph node & D.O.D. (9) & ++ & +++ \\
\hline 8 & $\mathrm{M} / 75$ & Lymph node & D.O.D. (5) & - & - \\
\hline 9 & $\mathrm{~F} / 65$ & Lymph node & D.O.D.(10) & +++ & +++ \\
\hline 10 & $\mathrm{~F} / 54$ & Lymph node & D.O.D.(37) & + & + \\
\hline 11 & $\mathrm{M} / 65$ & Lymph node & D.O.D.(17) & ++ & ++ \\
\hline 12 & M/69 & Lymph node & D.O.D. (4) & ++ & +++ \\
\hline 13 & $\mathrm{~F} / 54$ & Lymph node & D.O.D.(17) & - & +++ \\
\hline 14 & $\mathrm{M} / 71$ & Lymph node & D.O.D. (8) & ++ & +++ \\
\hline 15 & $\mathrm{~F} / 69$ & Lymph node & D.O.D.(19) & - & +++ \\
\hline 16 & $\mathrm{~F} / 69$ & Liver & n.i. & ++ & \\
\hline 17 & $\mathrm{~F} / 68$ & Liver & n.i. & + & \\
\hline 18 & $\mathrm{M} / 70$ & Liver & D.O.D. (4) & ++ & \\
\hline 19 & $\mathrm{~F} / 73$ & Liver & n.i. & ++ & \\
\hline 20 & $\mathrm{M} / 76$ & Liver & n.i. & ++ & \\
\hline 21 & M/68 & Liver & D.O.D. (2) & + & \\
\hline 22 & $\mathrm{~F} / 63$ & Liver & D.O.D. (3) & ++ & ++ \\
\hline 23 & $\mathrm{~F} / 56$ & Liver & D.O.D.(36) & + & \\
\hline 24 & M/73 & Liver & D.O.D. (3) & ++ & \\
\hline 25 & $\mathrm{~F} / 67$ & Liver & n.i. & + & \\
\hline 26 & $\mathrm{M} / 47$ & Liver & n.i. & + & \\
\hline 27 & $\mathrm{~F} / 61$ & Liver & Alive (29) & ++ & \\
\hline 28 & M/61 & Liver & D.O.D. (2) & ++ & \\
\hline 29 & $\mathrm{M} / 50$ & Liver & n.i. & ++ & \\
\hline
\end{tabular}

F, female; M, male; D.O.D., died of disease; n.i., no information available; -, negative staining in all tumor cells; +, positive staining in up to $20 \%$ of tumor cells; ++ , positive staining in up to $50 \%$ of tumor cells; +++ , positive staining in $>50 \%$ of tumor cells.

Fig. 1C. Besides expression in lymph node infiltrating tumor cells, L1CAM immunoreactivity was also detected in immune cells, a finding well corresponding to recent reports on L1CAM expression in dendritic cells (28). In addition, L1CAM expression was verified in all analysed liver metastases $(100 \%)$ comprising 5 cases with staining in up to $20 \%$ of tumor cells and 9 cases with positivity in up to $50 \%$ of tumor cells (Fig. 1D).

Statistical analysis of the data obtained with primary PDACs revealed no significant correlations between L1CAM expression and tumor grade, stage (data not shown) or survival (Fig. 1E). Nevertheless, there was a trend for a better survival for patients with tumors showing no or low L1CAM expression (up to $20 \%$ of tumor cells) vs. high L1CAM expression
( $>20 \%$ of tumor cells), resulting in median survival rates of 21.8 months (low L1CAM expression) and 15 months (high L1CAM expression).

Since we could already detect considerable L1CAM expression in chronic pancreatitis indicating that L1CAM upregulation is an early event in PDAC tumorigenesis (18), we also analysed L1CAM expression in PDAC precursor lesions, PanINs. As depicted in Fig. 2A-C, the examination of PanIN lesions (total $n=126$ ) showed a focal expression of mostly few, isolated cells in 3 of 47 PanIN 1A (6.4\%) and in 3 of 44 PanIN 1B (6.8\%). In PanIN 2, a focal L1CAM expression was seen in 7 of 20 lesions (35\%), and 3 of 15 PanIN 3 lesions $(20 \%)$ showed a focal $(n=1)$ or diffuse $(n=2)$ expression of L1CAM. A statistical comparison between low grade precursor 

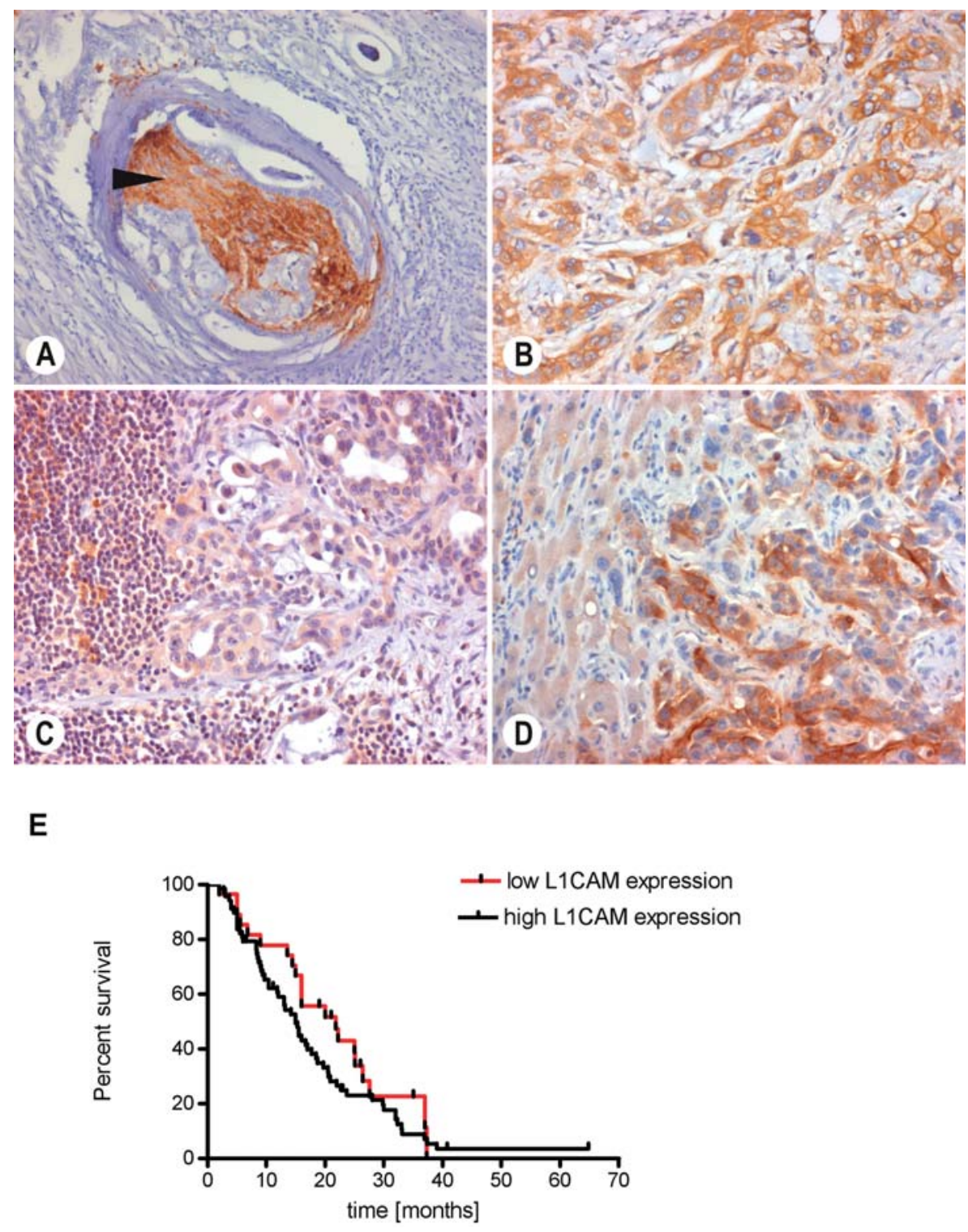

Figure 1 Immunohistochemical staining of L1CAM in primary and metastatic pancreatic ductal adenocarcinoma (PDAC). (A) Strong positivity for L1CAM in a nerve in primary PDAC (arrowhead), serving as internal control. (B) Strong L1CAM expression in primary PDAC. L1CAM expression in carcinoma cells in a (C) lymph node metastasis and (D) liver metastasis of PDAC patients. Original magnifications: x70 (A, C and D), x40 (B). (E) Correlation of L1CAM expression in tissues of primary PDAC and survival of 98 patients.

lesions (PanIN 1A/B) versus high grade lesions (PanIN 2 and 3) revealed a significant difference in L1CAM expression (p<0.001) (Fig. 2D).

\section{Discussion}

The prognosis of pancreatic ductal adenocarcinoma (PDAC) is extremely poor. At the onset of clinical symptoms, most PDACs have progressed to inoperable, locally and systemically advanced tumors. But also in cases of operability, radical resections (R0) are achieved in less than $25 \%$ of the patients (29), and many PDACs recur within the first years after surgical intervention. Furthermore, PDAC shows a pronounced resistance towards conventional radio- and/or chemotherapy. This urges the need for the identification of new, tumor specific molecular markers which may help to detect the tumors at an earlier stage of disease or may serve as target structures of a specific tumor therapy. In this study, based on a large series of 123 patients we show that L1CAM is expressed in the vast majority of PDAC (92.7\%), most frequently exhibiting a diffuse pattern of immunoreactivity within the tumors. This finding is in good accordance with previous results obtained in a smaller series of 20 PDAC, having revealed L1CAM expression in more than $80 \%$ of the tumors. However, in contrast to this, another study group reported L1CAM expression in only $2 \%$ of PDAC (22). One may speculate whether this discrepancy might be explained by different experimental protocols, including the criteria for evaluation of L1CAM positivity and for the selection of cases and/or eventual sampling errors in small probes. Nevertheless, several in vitro analyses indicate a role of L1CAM during the initiation and progression of PDAC, accounting for a chemoresistant and migratory phenotype of the tumor cells $(17,18)$. In the present series, the latter might be mirrored by a trend 


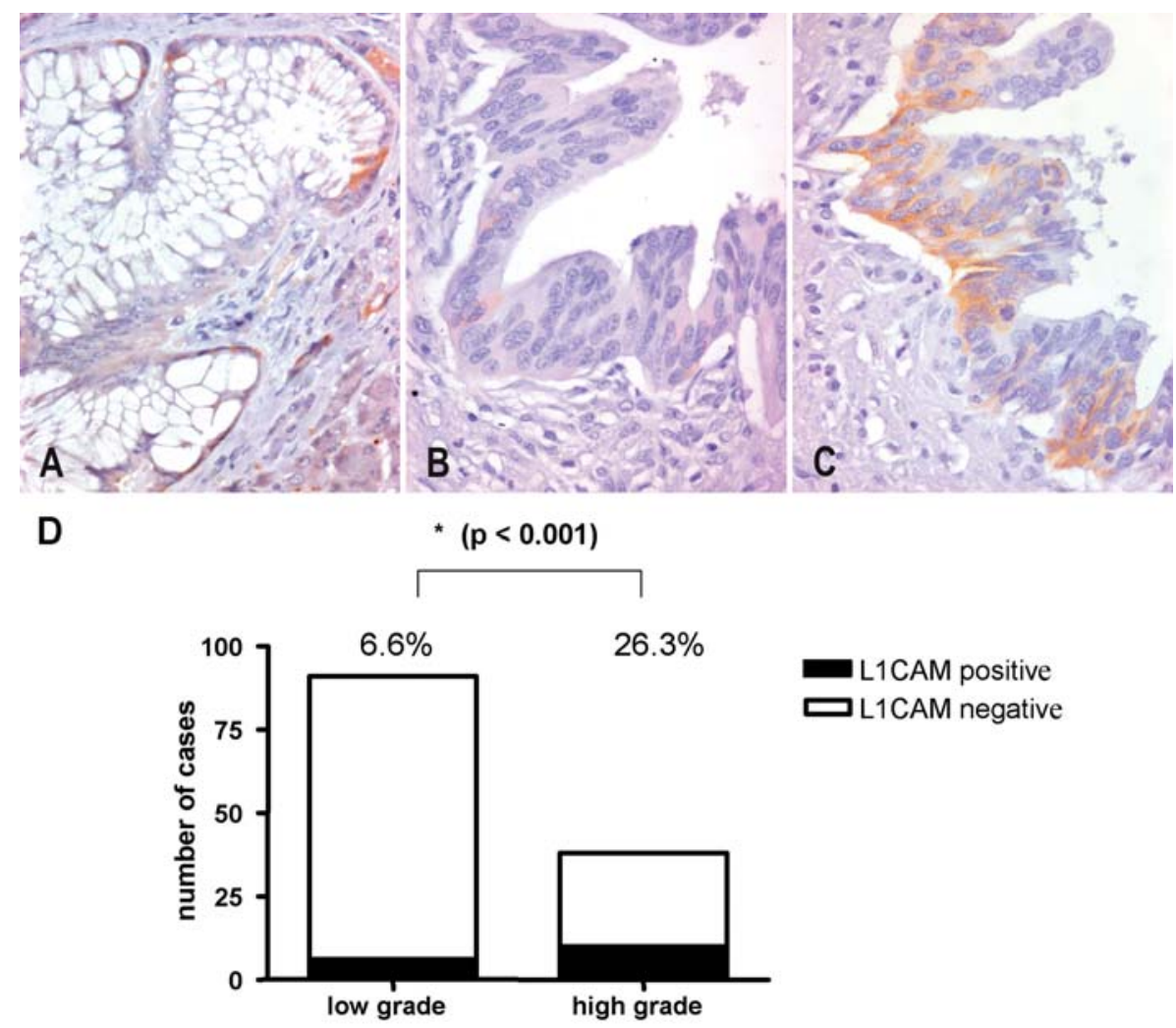

Figure 2 Immunohistochemical staining of L1CAM in pancreatic intraepithelial neoplasia (PanIN) grade 1A (A), grade 2 (B) and grade 3 (C). Original magnifications, x100. (D) L1CAM expression is significantly increased in high grade PanINs (PanIN 2 and 3) compared to low grade PanINs (PanIN 1A/B); " $\mathrm{p}<0.001$.

for a slightly better survival times in PDAC showing no or little ( $<20 \%$ of tumor cells) expression of L1CAM. However, most likely due to the fact that the prognosis of PDAC is generally very poor, this difference does not reach statistical significance. In the same line, no correlation between L1CAM expression and tumor grade and stage were found. However, as also reflected by the tumors of our series which exclusively presented at stage pT3 or pT4 and mostly displayed at least local metastatic spread, PDAC are only rarely found at early stages, and one might hypothesize that less pronounced L1CAM expression might be found at lower tumor stages. Apart from L1CAM expression in primary PDACs, we also identified L1CAM expression in $80 \%$ of the analysed lymph node metastases and in all liver metastases. These findings are in accordance with our recent results showing that L1CAM is an important mediator of cell migration of PDAC cells (18). Similar conclusions were drawn from studies with colorectal cancer patients (30) demonstrating L1CAM expression in micrometastases in lymph nodes and bone marrow.

Besides L1CAM expression in tissues, detection of soluble L1CAM in the serum of patients with ovarian and uterine carcinomas has been described (13). This proteolytically cleaved form of L1CAM could mainly be detected in the serum of patients with stage III-IV tumors, pointing to a potential use as prognostic marker (13). However, validation of these findings in a larger study is still pending. In contrast, cleavage of L1CAM seems not to be required for mediation of chemoresistance and migration of PDAC cells $(17,18)$ and soluble L1CAM could not be detected in serum samples of patients with PDAC (data not shown) which impairs its suitability for diagnostic purposes of PDAC patients.

In the present study, expression of L1CAM was also determined in PDAC precursor lesions, pancreatic intraepithelial neoplasia (PanIN). While PanIN 1A and PanIN 1B lesions seem to harbour only a rather small risk to transform into PDAC, malignant transformation is thought to expedite starting at PanIN 2 and PanIN 3 lesions (31). Correspondingly, in this study we demonstrate infrequent L1CAM expression in PanIN 1A and 1B lesions, while the frequency of L1CAM expression significantly increased in PanIN 2 and PanIN 3 suggesting a role of L1CAM in the early tumor initiating events. This finding further strengthens previous immunohistochemical findings in chronic pancreatitis specimens, revealing notable L1CAM expression in ductal structures surrounded by dense fibrotic tissue, indicating L1CAM induction in ductal epithelium already in a premalignant stage during the onset of desmoplasia upon persistent inflammation (18). Moreover, these data could be further supported by in vitro coculture experiments demonstrating that L1CAM expression in pancreatic ductal epithelial cells is induced by pancreatic myofibroblasts via TGF- 31 , a mechanism which is still operating in TGF- 31 responsive PDAC cells (18).

Since L1CAM is a cell surface molecule that is overexpressed in the vast majority of carcinoma cells in primary tumors and metastases, but only rarely expressed in normal tissues, its use as a therapeutical target appears to be reasonable. L1CAM has been implicated in various tumor-associated processes such as cell migration, metastasis, proliferation and 
apoptosis resistance, thus targeting of L1CAM could interfere with tumor progression at numerous points, thereby enhancing anti-tumor responses. Indeed, blocking or knock-down of L1CAM expression in various tumors decreased cell migration $(9,18)$, increased the apoptotic response towards cytostatic drugs in vitro $(17,32)$, and suppressed tumor growth in vivo $(16,33)$. In the latter context, it has been shown that a subpopulation of glioma cells, defined as $\mathrm{CD} 133^{+}$tumor-initiating or cancer stem cells, is also characterised by the expression of L1CAM (33). Knock-down of L1CAM expression in these tumor cells reduced tumor growth and increased survival of tumor-bearing nude mice (33). These data suggest that therapeutic targeting of L1CAM will allow the elimination of tumor-initiating, therapy resistant cells. Most notably, combinations of L1CAM targeting strategies with other therapeutic options (e.g., chemo- or radiotherapy, labelling of L1CAM antibodies with radioactive substances) appear to be most promising because of the expected synergistic and/or additive effects, e.g., as it has been already shown for the chemosensitizing effect of L1CAM blockade in PDAC cells (17).

\section{Acknowledgements}

The authors thank Sabine Roggenbrodt and Vesna Vukovic for excellent technical support. This study was supported by the German Research Society DFG SE-1831/2-1 and the cluster of excellence Inflammation at Interfaces (S.S.M.) and the Tumorzentrum Heidelberg/Mannheim (F.B.)

\section{References}

1. Lockhart AC, Rothenberg ML and Berlin JD: Treatment for pancreatic cancer: current therapy and continued progress. Gastroenterology 128: 1642-1654, 2005.

2. Schneider G, Siveke JT, Eckel F, et al: Pancreatic cancer: basic and clinical aspects. Gastroenterology 128: 1606-1625, 2005.

3. Jemal A, Siegel R, Ward E, et al: Cancer statistics, 2007. CA Cancer J Clin 57: 43-66, 2007.

4. Müerköster S, Wegehenkel K, Arlt A, et al: Tumor stroma interactions induce chemoresistance in pancreatic ductal carcinoma cells involving increased secretion and paracrine effects of nitric oxide and interleukin-1beta. Cancer Res 64 1331-1337, 2004.

5. Sebens Müerköster S, Lust J, Arlt A, et al: Acquired chemoresistance in pancreatic carcinoma cells: induced secretion of IL-1beta and NO lead to inactivation of caspases. Oncogene 25: 3973-3981, 2006.

6. Moos M, Tacke R, Scherer H, et al: Neural adhesion molecule L1 as a member of the immunoglobulin superfamily with binding domains similar to fibronectin. Nature 334: 701-703, 1988.

7. Brummendorf T, Kenwrick S and Rathjen FG: Neural cell recognition molecule L1: from cell biology to human hereditary brain malformations. Curr Opin Neurobio 8: 87-97, 1998.

8. Gast D, Riedle S, Schabath H, et al: L1 augments cell migration and tumor growth but not beta3 integrin expression in ovarian carcinomas. Int J Cancer 115: 658-665, 2005.

9. Gavert N, Conacci-Sorrell M, Gast D, et al: L1, a novel target of beta-catenin signaling, transforms cells and is expressed at the invasive front of colon cancers. J Cell Biol 168: 633-642, 2005.

10. Izumoto $\mathrm{S}$, Ohnishi T, Arita N, et al: Gene expression of neural cell adhesion molecule L1 in malignant gliomas and biological significance of L1 in glioma invasion. Cancer Res 56: 1440-1444, 1996.

11. Kaifi JT, Strelow A, Schurr PG, et al: L1 (CD171) is highly expressed in gastrointestinal stromal tumors. Mod Pathol 19: 399-406, 2006.

12. Meier F, Busch S, Gast D, et al: The adhesion molecule L1 (CD171) promotes melanoma progression. Int J Cancer 119 : $549-555,2006$
13. Fogel M, Gutwein P, Mechtersheimer S, et al: L1 expression as a predictor of progression and survival in patients with uterine and ovarian carcinomas. Lancet 362: 869-875, 2003.

14. Maretzky T, Schulte M, Ludwig A, et al: L1 is sequentially processed by two differently activated metalloproteases and presenilin/gamma-secretase and regulates neural cell adhesion, cell migration and neurite outgrowth. Mol Cell Biol 25: 9040-9053, 2005.

15. Mechtersheimer S, Gutwein P, Agmon-Levin N, et al: Ectodomain shedding of L1 adhesion molecule promotes cell migration by autocrine binding to integrins. J Cell Biol 155: 661-673, 2001.

16. Arlt MJ, Novak-Hofer I, Gast D, et al: Efficient inhibition of intra-peritoneal tumor growth and dissemination of human ovarian carcinoma cells in nude mice by anti-L1-cell adhesion molecule monoclonal antibody treatment. Cancer Res 66: 936-943, 2006.

17. Sebens Müerköster S, Werbing V, Sipos B, et al: Drug-induced expression of the cellular adhesion molecule L1CAM confers anti-apoptotic protection and chemoresistance in pancreatic ductal adenocarcinoma cells. Oncogene 26: 2759-2768, 2007.

18. Geismann C, Morscheck M, Koch D, et al: Up-regulation of L1CAM in pancreatic duct cells is transforming growth factor beta1- and slug-dependent: role in malignant transformation of pancreatic cancer. Cancer Res 69: 4517-4526, 2009.

19. Huszar M, Moldenhauer G, Gschwend V, et al: Expression profile analysis in multiple human tumors identifies L1 (CD171) as a molecular marker for differential diagnosis and targeted therapy. Human Pathol 37: 1000-1008, 2006.

20. Kaifi JT, Zinnkann U, Yekebas EF, et al: L1 is a potential marker for poorly-differentiated pancreatic neuroendocrine carcinoma. World J Gastroenterol 12: 94-98, 2006.

21. Bergmann F, Moldenhauer G, Herpel E, et al: Expression of L1CAM, COX-2, EGFR, c-KIT and Her2/neu in anaplastic pancreatic cancer: putative therapeutic targets? Histopathology 56: 440-448, 2010.

22. Kaifi JT, Heidtmann S, Schurr PG, et al: Absence of L1 in pancreatic masses distinguishes adenocarcinoma from poorly differentiated neuroendocrine carcinomas. Anticancer Res 26: 1167-1170, 2006.

23. Sebens Müerköster S, Kötteritzsch J, Geismann C, et al: $\alpha 5$ integrin is crucial for L1CAM-mediated chemoresistance in pancreatic adenocarcinoma. Int J Oncol 34: 243-253, 2009.

24. Klöppel G, Hruban RH, Longnecker DS, Adler G, Kern SE and Partanen TJ: Ductal adenocarcinoma of the pancreas. In: World Health Organization of Tumous. Pathology and Genetics of Tumours of the Digestive System. Hamilton SR and Aaltonen LA (eds). IARC Press, Lyon, 2000.

25. Sitek B, Sipos B, Alkatout I, et al: Analysis of the pancreatic tumor progression by a quantitative proteomic approach and immunhistochemical validation. J Proteome Res 8: 1647-1656, 2009.

26. Bergmann F, Ceyhan GO, Rieker RJ, et al: Fundamental differences in the neural invasion behavior of pancreatic endocrine tumors: relevance for local recurrence rates? Human Pathol 40: 50-57, 2009.

27. Bergmann F, Hackert T, Mechtersheimer G, et al: Differential diagnosis of non-epithelial tumors of the pancreas: malignant non-epithelial pancreatic tumor with focal pigmentation. Virchows Arch 444: 190-193, 2004.

28. Maddaluno L, Verbrugge SE, Martinoli C, et al: The adhesion molecule L1 regulates transendothelial migration and trafficking of dendritic cells. J Exp Med 209: 623-635, 2009.

29. Esposito I, Kleeff J, Bergmann F, et al: Most pancreatic cancer resections are R1 resections. Ann Surg Oncol 15: 1651-1660, 2008.

30. Kaifi JT, Reichelt U, Quaas A, et al: L1 is associated with micrometastatic spread and poor outcome in colorectal cancer. Modern Path 20: 1183-1190, 2007.

31. Sipos B, Frank S, Gress T, Hahn S and Klöppel G: Pancreatic intraepithelial neoplasia revisited and updated. Pancreatology 9: 45-54, 2009.

32. Stoeck A, Gast D and Sanderson MP: L1-CAM in a membranebound or soluble form augments protection from apoptosis in ovarian carcinoma cells. Gynecol Oncol 104: 461-469, 2007.

33. Bao S, Wu Q, Li Z, et al: Targeting cancer stem cells through L1CAM suppresses glioma growth. Cancer Res 68: 6043-6048, 2008. 